Article

\title{
The Existence of Nontrivial Solution for a Class of Kirchhoff-Type Equation of General Convolution Nonlinearity without Any Growth Conditions
}

\author{
Li Zhou ${ }^{1,2}$ and Chuanxi Zhu ${ }^{1, *}$ \\ 1 Department of Mathematics, Nanchang University, Nanchang 330031, China; zhouli8869@gmail.com \\ 2 Department of Basic Discipline, Nanchang JiaoTong Institute, Nanchang 330031, China \\ * Correspondence: zhuchuanxi@ncu.edu.cn
}

check for

updates

Citation: Zhou, L.; Zhu, C. The Existence of Nontrivial Solution for a Class of Kirchhoff-Type Equation of General Convolution Nonlinearity without Any Growth Conditions. Axioms 2021, 10, 163. https:// doi.org/10.3390/axioms10030163

Academic Editors: Feliz Manuel Minhós and Chris Goodrich

Received: 25 April 2021 Accepted: 13 July 2021

Published: 27 July 2021

Publisher's Note: MDPI stays neutral with regard to jurisdictional claims in published maps and institutional affiliations.

Copyright: (c) 2021 by the authors. Licensee MDPI, Basel, Switzerland. This article is an open access article distributed under the terms and conditions of the Creative Commons Attribution (CC BY) license (https:/ / creativecommons.org/licenses/by/ $4.0 /)$.
Abstract: In this paper, we consider the following Kirchhoff-type equation: $\left\{\begin{array}{l}-\left(a+b \int_{\mathbb{R}^{N}}|\nabla u|^{2} \mathrm{~d} x\right) \Delta u+V(x) u=\left(I_{\alpha} * F(u)\right) f(u)+\lambda g(u), \text { in } \mathbb{R}^{N}, \\ u \in H^{1}\left(\mathbb{R}^{N}\right),\end{array}\right.$ $N \geq 3, V: \mathbb{R}^{N} \rightarrow \mathbb{R}$ is a potential function and $I_{\alpha}$ is a Riesz potential of order $\alpha \in(N-2, N)$. Under certain assumptions on $V(x), f(u)$ and $g(u)$, we prove that the equation has at least one nontrivial solution by variational methods.

Keywords: Kirchhoff equation; no growth conditions; cutoff function

MSC: 35J60; 35J35; 35R11; 35A15

\section{Introduction}

In this article, we study the following Kirchhoff-type equation:

$$
\left\{\begin{array}{l}
-\left(a+b \int_{\mathbb{R}^{N}}|\nabla u|^{2} \mathrm{~d} x\right) \Delta u+V(x) u=\left(I_{\alpha} * F(u)\right) f(u)+\lambda g(u), \text { in } \mathbb{R}^{N}, \\
u \in H^{1}\left(\mathbb{R}^{N}\right),
\end{array}\right.
$$

where $a>0, b \geq 0, \lambda>0, \alpha \in(N-2, N), N \geq 3, F(t)=\int_{0}^{t} f(s) \mathrm{d} s$ and $I_{\alpha}$ is a Riesz potential for which its order is $\alpha \in(N-2, N)$. Here, $I_{\alpha}$ is defined by $I_{\alpha}=\frac{\Gamma\left(\frac{N-\alpha}{2}\right)}{\Gamma\left(\frac{\alpha}{2}\right) \pi^{\frac{N}{2}} 2^{\alpha}|x|^{N-\alpha}}$. Moreover, $V(x): \mathbb{R}^{N} \rightarrow \mathbb{R}$ is a potential function satisfying the following.

(V) $\inf _{x \in \mathbb{R}^{N}} V(x)=V_{0}>0$ and for any $M>0$, there exist $r>0$ such that:

$$
\lim _{|y| \rightarrow+\infty} \operatorname{meas}\left\{x \in \mathbb{R}^{N}:|x-y| \leq r, V(x) \leq M\right\}=0
$$

Additionally, we suppose that the function $f \in C^{1}(\mathbb{R}, \mathbb{R})$ verifies:

(f1) $f(t)=o\left(t \frac{\alpha}{N}\right)$ as $t \rightarrow 0$;

(f2) $\lim _{|t| \rightarrow+\infty} \frac{f(t)}{t^{\frac{\alpha+2}{N-2}}}=0$;

(f3) $\frac{f(t)}{t}$ is increasing on $(0,+\infty)$ and decreasing on $(-\infty, 0)$;

$(f 4) f(t)$ is increasing on $\mathbb{R}$.

Furthermore, we assume that the function $g \in C(\mathbb{R}, \mathbb{R})$ satisfies the following.

$(g 1) g(t)=o(t)$ as $t \rightarrow 0$;

(g2) $\lim _{|t| \rightarrow+\infty} \frac{g(t)}{t}=+\infty$.

It is worth mentioning that, here, $g$ maybe critical or supercritical.

In the past decades, many scholars have studied the existence of nontrivial solutions for the Kirchhoff-type problem: 


$$
\left\{\begin{array}{l}
-\left(a+b \int_{\mathbb{R}^{3}}|\nabla u|^{2} \mathrm{~d} x\right) \Delta u+V(x) u=g(x, u), \text { in } \mathbb{R}^{3}, \\
u \in H^{1}\left(\mathbb{R}^{3}\right),
\end{array}\right.
$$

where $a>0, b \geq 0, V: \mathbb{R}^{3} \rightarrow \mathbb{R}$ is a potential function and $g \in C\left(\mathbb{R}^{3} \times \mathbb{R}, \mathbb{R}\right)$. Problem (2) is a nonlocal problem due to the presence of the term $b \int_{\mathbb{R}^{3}}|\nabla u|^{2} \mathrm{~d} x$, which causes some mathematical difficulties but, at the same time, renders the research problem particular interesting. This problem has a profound and interesting physical context. Indeed, if we set $V(x)=0$ and replace $\mathbb{R}^{3}$ by a bounded domain $\Omega \subset \mathbb{R}^{3}$ in (2), then we obtain the following Kirchhoff Dirichlet problem.

$$
\begin{cases}-\left(a+b \int_{\mathbb{R}^{3}}|\nabla u|^{2} \mathrm{~d} x\right) \Delta u=g(x, u), & x \in \Omega, \\ u=0 & x \in \partial \Omega .\end{cases}
$$

It is related to the stationary analogue of the equation, as shown as follows:

$$
\rho \frac{\partial^{2} u}{\partial t^{2}}-\left(\frac{\rho_{0}}{h}+\frac{E}{2 L} \int_{0}^{L}\left|\frac{\partial u}{\partial x}\right| \mathrm{d} x\right) \frac{\partial^{2} u}{\partial x^{2}}=0
$$

which was proposed by G.Kirchhoff as an extension of classical D'Alembert's wave equations for the free vibration of elastic strings. Kirchhoff's model takes into account the changes in the length of the string produced by transverse vibrations. J. L. Lions soon completed the pioneer work. He introduced a functional analysis approach. Since then, Kirchhoff equations have attracted the attention of many researchers. The works include Readers can see [1-11] and the references therein. Unfortunately, most of the studies assume that the growth condition and the Ambrosetti-Rabinowtiz condition are satisfied. On the bright side, in [4], Guo studied the following Kirchhoff-type problem.

$$
\left\{\begin{array}{l}
-\left(a+b \int_{\mathbb{R}^{3}}|\nabla u|^{2} \mathrm{~d} x\right) \Delta u+V(x) u=f(u), \text { in } \mathbb{R}^{3}, \\
u \in H^{1}\left(\mathbb{R}^{3}\right) .
\end{array}\right.
$$

He proved the existence of a positive ground state solution to (2) without any (A-R) type condition. Furthermore, in [10], the authors obtained the existence of a nontrivial solution for the following Kirchhoff-type equation.

$$
\left\{\begin{array}{l}
-\left(a+b \int_{\mathbb{R}^{3}}|\nabla u|^{2} \mathrm{~d} x\right) \Delta u+V(x) u=|u|^{p-2} u+\lambda f(u), \text { in } \mathbb{R}^{3}, \\
u \in H^{1}\left(\mathbb{R}^{3}\right) .
\end{array}\right.
$$

In [10], there is no Ambrosetti-Rabinowtiz and no growth condition. Moreover, their conclusion holds for general supercritical nonlinearity.

On the other hand, when $a=1, b=0$ and $f=0$, the Equation (1) reduces to:

$$
-\Delta u+V(x) u=\left(I_{\alpha} *|u|^{p}\right)|u|^{p-2} u,
$$

which is called nonlinear Choquard type equation. Its physical background can be found in [12] and the references therein. Furthermore, readers can investigate [6,13-20] for recent achievements.

Motivated by the works mentioned above, especially by [10,21,22], we consider the combination of the two types of Equations (4) and (5) and extend to the general convolution case in $\mathbb{R}^{N}$. In our paper, we obtain the nontrivial solution of Equation (1).

The main outcome of our investigation is as follows.

Theorem 1. If $(V),(f 1)-(f 4)$ and $(g 1),(g 2)$ hold, then problem (1) has at least one nontrivial solution for $\lambda$ small.

For the convenience of expression, hereafter, we use the following notations: 
- $\quad X:=\left\{u \in H^{1}\left(\mathbb{R}^{N}\right): \int_{\mathbb{R}^{N}} V(x) u^{2} \mathrm{~d} x<\infty\right\}$ is equipped with an equivalent norm $\|u\|=\left[\int_{\mathbb{R}^{N}}\left(a|\nabla u|^{2}+V(x) u^{2}\right) \mathrm{d} x\right]^{\frac{1}{2}}$

- $\quad L^{s}\left(\mathbb{R}^{N}\right)(1 \leq s \leq \infty)$ denotes the Lebesgue space with the norm $|u|_{s}=\left(\int_{\mathbb{R}^{N}}|u|^{s} \mathrm{~d} x\right)^{1 / s}$;

- For any $u \in H^{1}\left(\mathbb{R}^{N}\right) \backslash\{0\}, u_{t}$ is denoted as follows.

$$
u_{t}= \begin{cases}0, & t=0 \\ \sqrt{t} u\left(\frac{x}{t}\right), & t>0,\end{cases}
$$

- For any $x \in \mathbb{R}^{N}$ and $r>0, B_{r}(x):=\left\{y \in \mathbb{R}^{N}:|y-x|<r\right\}$;

- $\quad C, C_{1}, C_{2}, \ldots$ represent positive constants that are possibly different in different lines.

Remark 1. According to the condition (V) and [23], $X \hookrightarrow L^{r}\left(\mathbb{R}^{N}\right)$ is compact, $r \in\left[2,2^{*}\right)$, where $2^{*}=\frac{2 N}{N-2}$ if $N \geq 3$ and $2^{*}=\infty$ if $N=1$ or 2 .

\section{Preliminaries}

In this section, we will provide the revised functional and some lemmas. Notice that there is no growth condition and no Ambrosetti-Rabinowitz condition and so we require the cutoff function.

According to $(g 2)$, we have $g(M)>0$ as $M>0$ large and so we define the cutoff function as follows.

$$
h_{M}(t)= \begin{cases}g(t), & 0<t \leq M, \\ C_{M} t^{p-1}, & t>M, \\ 0, & t \leq 0 .\end{cases}
$$

Here $C_{M}=\frac{g(M)}{M^{p-1}}, 2<p<2^{*}$. Since $g \in C(\mathbb{R}, \mathbb{R}), h_{M}$ is also continuous. Moreover, by $(g 1), h_{M}$ satisfies the following.

$(h 1) h_{M}(t)=o(t)$ as $t \rightarrow 0$;

(h2) $\lim _{t \rightarrow+\infty} \frac{H_{M}(t)}{t^{4}}=+\infty$, where $H_{M}(t)=\int_{0}^{t} h_{M}(s) \mathrm{d} s$;

(h3) $\left|h_{M}(t)\right| \leq C_{M}^{\prime}|t|+C_{M}|t|^{p-1}$, where $C_{M}^{\prime}=\max _{t \in[0, M]} \frac{|g(t)|}{t}$;

(h4) there exist $\theta=\theta(M)>0$ such that $t h_{M}(t)-4 H_{M}(t) \geq-\theta t^{2}, t \geq 0$.

Next, we first consider the following revised problem.

$$
-\left(a+b \int_{\mathbb{R}^{N}}|\nabla u|^{2} \mathrm{~d} x\right) \Delta u+V(x) u=\left(I_{\alpha} * F(u)\right) f(u)+\lambda h_{M}(u), x \text { in } \mathbb{R}^{N},
$$

Problem (6) has a variational structure, i.e., the critical points of the functional $I_{\lambda}^{M}$ : $X \rightarrow \mathbb{R}$ is defined as follows:

$$
\begin{aligned}
I_{\lambda}^{M}(u) & =\frac{1}{2} \int_{\mathbb{R}^{N}}\left[a|\nabla u|^{2}+V(x) u^{2}\right] \mathrm{d} x+\frac{b}{4}\left(\int_{\mathbb{R}^{N}}|\nabla u|^{2} \mathrm{~d} x\right)^{2} \\
& -\frac{1}{2} \int_{\mathbb{R}^{N}}\left(I_{\alpha} * F(u)\right) F(u) \mathrm{d} x-\lambda \int_{\mathbb{R}^{N}} H_{M}(u) \mathrm{d} x, u \in X
\end{aligned}
$$

which are weak solutions of problem (6).

It is obvious that $I_{\lambda}^{M}$ is of class $C^{1}$ and the following is the case.

$$
\begin{aligned}
\left\langle\left(I_{\lambda}^{M}\right)^{\prime}(u), v\right\rangle & =\int_{\mathbb{R}^{N}}[a \nabla u \nabla v+V(x) u v] \mathrm{d} x+b \int_{\mathbb{R}^{N}}|\nabla u|^{2} \mathrm{~d} x \int_{\mathbb{R}^{N}} \nabla u \nabla v \mathrm{~d} x \\
& -\int_{\mathbb{R}^{N}}\left(I_{\alpha} * F(u)\right) f(u) v \mathrm{~d} x-\lambda \int_{\mathbb{R}^{N}} h_{M}(u) v \mathrm{~d} x .
\end{aligned}
$$

Lemma 1. Assume (f1)-(f4) are fulfilled, then we have: 
(1) for all $\varepsilon>0$, there is a $C_{\varepsilon}>0$ such that $|f(t)| \leq \varepsilon|t|^{\frac{\alpha}{N}}+C_{\varepsilon}|t|^{\frac{\alpha+2}{N-2}}$ and $|F(t)| \leq$ $\varepsilon|t|^{\frac{N+\alpha}{N}}+C_{\varepsilon}|t|^{\frac{N+\alpha}{N-2}}$;

(2) for all $\varepsilon>0$, there is a $C_{\varepsilon}>0$ such that for every $p \in\left(2,2^{*}\right),|F(t)| \leq \varepsilon\left(|t|^{\frac{N+\alpha}{N}}+\right.$ $\left.|t|^{\frac{N+\alpha}{N-2}}\right)+C_{\varepsilon}|t|^{\frac{p(N+\alpha)}{2 N}}$, and $|F(t)|^{\frac{2 N}{N+\alpha}} \leq \varepsilon\left(|t|^{2}+|t|^{\frac{2 N}{N-2}}\right)+C_{\varepsilon}|t|^{p}$;

(3) for any $s \neq 0, s f(s)>2 F(s)$ and $F(s)>0$.

Proof. One can easily obtain the results by elementary calculation.

Lemma 2. (Hardy-Littlewood-Sobolev inequality [24]). Let $0<\alpha<N, p, q>1$ and $1 \leq r<$ $s<\infty$ be such that

$$
\frac{1}{p}+\frac{1}{q}=1+\frac{\alpha}{N}, \frac{1}{r}-\frac{1}{s}=\frac{\alpha}{N} .
$$

(1) For any $f \in L^{p}\left(\mathbb{R}^{N}\right)$ and $g \in L^{q}\left(\mathbb{R}^{N}\right)$, one has

$$
\left|\int_{\mathbb{R}^{N}} \int_{\mathbb{R}^{N}} \frac{f(x) g(y)}{|x-y|^{N-\alpha}} d x d y\right| \leq C(N, \alpha, p)\|f\|_{L^{p}\left(\mathbb{R}^{N}\right)}\|g\|_{L^{q}\left(\mathbb{R}^{N}\right)} .
$$

(2) For any $f \in L^{r}\left(\mathbb{R}^{N}\right)$ one has

$$
\left\|\frac{1}{|\cdot|^{N-\alpha}} * f\right\|_{L^{s}\left(\mathbb{R}^{N}\right)} \leq C(N, \alpha, r)\|f\|_{L^{r}\left(\mathbb{R}^{N}\right)} .
$$

Remark 2. By Lemma 1 (1), Lemma 2 (1) and the Sobolev imbedding theorem, we can obtain the following.

$$
\begin{aligned}
& \mid \int_{\mathbb{R}^{N}}\left.\left(I_{\alpha} * F(u)\right) F(u) d x|\leq C| F(u)\right|_{\frac{2 N}{N+\alpha}} ^{2} \\
& \quad \leq C\left[\int_{\mathbb{R}^{N}}\left(|u|^{\frac{N+\alpha}{N}}+|u|^{\frac{N+\alpha}{N-2}}\right)^{\frac{(2 N)}{N+\alpha}} d x\right]^{\frac{N+\alpha}{N}} \\
& \quad \leq C\left[\int_{\mathbb{R}^{N}}\left(|u|^{2}+|u|^{\frac{2 N}{N-2}}\right) d x\right]^{\frac{N+\alpha}{N}} \\
& \leq C\left(\|u\|^{\frac{2 N+2 \alpha}{N}}+\|u\|^{\frac{2 N+2 \alpha}{N-2}}\right)
\end{aligned}
$$

\section{Variational Formulation}

In this section, we will prove the following results.

Lemma 3. $I_{\lambda}^{M}(u)$ satisfies (PS) condition.

Proof. Let $\left\{u_{n}\right\}$ be $(P S)_{c}$ sequence of $I_{\lambda}^{M}(u)$. Then by $(h 4)$ we have the following.

$$
\begin{aligned}
c+o(1)\left\|u_{n}\right\| & \geq 4 I_{\lambda}^{M}\left(u_{n}\right)-\left\langle\left(I_{\lambda}^{M}\right)^{\prime}\left(u_{n}\right), u_{n}\right\rangle \\
& =\int_{\mathbb{R}^{N}}\left[a\left|\nabla u_{n}\right|^{2}+V(x)\left|u_{n}\right|^{2}\right] \mathrm{d} x+\int_{\mathbb{R}^{N}}\left(I_{\alpha} * F\left(u_{n}\right)\right)\left[f\left(u_{n}\right) u_{n}-2 F\left(u_{n}\right)\right] \mathrm{d} x+\lambda \int_{\mathbb{R}^{N}}\left[h_{M}\left(u_{n}\right) u_{n}-4 H_{M}\left(u_{n}\right)\right] \mathrm{d} x \\
& \geq \int_{\mathbb{R}^{N}}\left[a\left|\nabla u_{n}\right|^{2}+V(x)\left|u_{n}\right|^{2}\right] \mathrm{d} x+\lambda \int_{\mathbb{N}^{N}}\left[h_{M}\left(u_{n}\right) u_{n}-4 H_{M}\left(u_{n}\right)\right] \mathrm{d} x \\
& \geq \int_{\mathbb{R}^{N}}\left[a\left|\nabla u_{n}\right|^{2}+V(x)\left|u_{n}\right|^{2}\right] \mathrm{d} x-\lambda \int_{\mathbb{R}^{N}} \theta\left|u_{n}^{+}\right|^{2} \mathrm{~d} x .
\end{aligned}
$$

Here $u_{n}^{+}=\max \left\{u_{n}, 0\right\}, u_{n}^{-}=\min \left\{u_{n}, 0\right\}$ and $u_{n}=u_{n}^{+}+u_{n}^{-}$. If $\left\{u_{n}\right\}$ is unbounded, i.e., $\left\|u_{n}\right\| \rightarrow \infty$. Let $v_{n}=\frac{u_{n}}{\left\|u_{n}\right\|}$, then $v_{n}=v_{n}^{+}+v_{n}^{-}$and $\left\|v_{n}\right\|=1$. Thus, we can obtain the fact that there exist a $v \in X$ such that: 


$$
\left\{\begin{array}{l}
v_{n} \rightarrow v \text { in } X \\
v_{n} \rightarrow v \text { in } L^{s}\left(\mathbb{R}^{N}\right), \forall s \in\left[2,2^{*}\right) \\
v_{n} \rightarrow v \text { a.e. on } \mathbb{R}^{N}
\end{array}\right.
$$

and

$$
\left\{\begin{array}{l}
v_{n}^{+} \rightarrow v^{+} \text {in } X, \\
v_{n}^{+} \rightarrow v^{+} \text {in } L^{s}\left(\mathbb{R}^{N}\right), \forall s \in\left[2,2^{*}\right) \\
v_{n}^{+} \rightarrow v^{+} \text {a.e. on } \mathbb{R}^{N} .
\end{array}\right.
$$

By (10) we have the following.

$$
o(1) \geq\left\|v_{n}\right\|^{2}-\lambda \theta \int_{\mathbb{R}^{N}}\left|v_{n}^{+}\right|^{2} \mathrm{~d} x=1-\lambda \theta \int_{\mathbb{R}^{N}}\left|v^{+}\right|^{2} \mathrm{~d} x+o(1) .
$$

Therefore $v^{+} \neq 0$. By $(10)$ and $\left(I_{\lambda}^{M}\right)^{\prime}\left(u_{n}\right) \rightarrow 0$, we have the following.

$$
\begin{aligned}
o(1)= & \frac{\left\langle\left(I_{\lambda}^{M}\right)^{\prime}\left(u_{n}\right), u_{n}\right\rangle}{\left\|u_{n}\right\|^{4}} \\
& =o(1)+b\left(\int_{\mathbb{R}^{N}}\left|\nabla v_{n}\right|^{2} \mathrm{~d} x\right)^{2}-\left\|u_{n}\right\|^{-4} \int_{\mathbb{R}^{N}}\left(I_{\alpha} * F\left(u_{n}\right)\right) f\left(u_{n}\right) u_{n} \mathrm{~d} x-\lambda \int_{\mathbb{R}^{N}} \frac{h_{M}\left(u_{n}^{+}\right) u_{n}^{+}}{\left|u_{n}^{+}\right|^{4}}\left|v_{n}^{+}\right|^{4} \\
& \leq o(1)+b\left(\int_{\mathbb{R}^{N}}\left|\nabla v_{n}\right|^{2} \mathrm{~d} x\right)^{2}-\lambda \int_{\mathbb{R}^{N}} \frac{h_{M}\left(u_{n}^{+}\right) u_{n}^{+}}{\left|u_{n}^{+}\right|^{4}}\left|v_{n}^{+}\right|^{4} .
\end{aligned}
$$

It is obvious that $u_{n}^{+}=v_{n}^{+}\left\|u_{n}\right\| \rightarrow+\infty$ a.e. $x \in\left\{x \in \mathbb{R}^{N}: v_{n}^{+}(x) \neq 0\right\}$. Together with (11) and (h2), we have $0 \leq-\infty$, which is a contradiction. Therefore, $\left\{u_{n}\right\}$ is bounded in $X$. Then, by standard methods we can obtain the convergence of $\left\{u_{n}\right\}$.

Lemma 4. The functional $I_{\lambda}^{M}$ possesses the mountain-pass geometry, i.e.:

(1) There exist $\rho, \delta>0$ such that $I_{\lambda}^{M} \geq \delta$ for all $\|u\|=\rho$;

(2) There exist $e \in H^{1}\left(\mathbb{R}^{3}\right)$ such that $\|e\|>\rho$ and $I_{\lambda}^{M}(e)<0$.

Proof. (1) By (h3) and Lemma 1, we have the following.

$$
I_{\lambda}^{M}(u) \geq C_{1}\|u\|^{2}-C_{2}\left(\|u\|^{\frac{2 N+2 \alpha}{N}}+\|u\|^{\frac{2 N+2 \alpha}{N-2}}\right)-C_{\varepsilon}\|u\|^{p} .
$$

Thus, there exist $\rho, \delta>0$ such that $I_{\lambda}^{M} \geq \delta$ for all $\|u\|=\rho>0$ is small enough.

(2) We freely choose $u \in C_{0}^{\infty}\left(\mathbb{R}^{3}\right)$, then we can obtain:

$$
\begin{aligned}
I_{\lambda}^{M}\left(u_{t}\right) & =\frac{a t^{N-1}}{2} \int_{\mathbb{R}^{N}}|\nabla u|^{2} \mathrm{~d} x+\frac{t^{N+1}}{2} \int_{\mathbb{R}^{N}} V(x) u^{2} \mathrm{~d} x+\frac{b t^{2 N-2}}{4}\left(\int_{\mathbb{R}^{N}}|\nabla u|^{2} \mathrm{~d} x\right)^{2} \\
& -\frac{t^{N+\alpha}}{2} \int_{\mathbb{R}^{N}}\left(I_{\alpha} * F(\sqrt{t} u)\right) F(\sqrt{t} u) \mathrm{d} x-\lambda \int_{\mathbb{R}^{N}} H_{M}(\sqrt{t} u) \mathrm{d} x \rightarrow-\infty,
\end{aligned}
$$

as $t \rightarrow+\infty$.

Note the following.

$$
\left\|u_{t}\right\|^{2}=a t^{N-1} \int_{\mathbb{R}^{N}}|\nabla u|^{2} \mathrm{~d} x+t^{N+1} \int_{\mathbb{R}^{N}} V_{\infty} u^{2} \mathrm{~d} x .
$$

Thus, in taking $e=t_{0} u$ with $t_{0}>0$ large, we have $\|e\|>\rho$ and $I_{\lambda}^{M}(e)<0$.

Remark 3. Now we can define the mountain-pass level of $I_{\lambda}^{M}$ : 


$$
c_{\lambda}^{M}=\inf _{\gamma \in \Gamma \in[0,1]} \max _{\lambda}^{M}(\gamma(t))>0,
$$

where: $\Gamma=\left\{\gamma \in C([0,1], X): \gamma(0)=0, I_{\lambda}^{M}(\gamma(1))<0\right\}$. Then, according to [25] and Lemma 3, $I_{\lambda}^{M}$ has a critical point $u_{\lambda}$ with $I_{\lambda}^{M}\left(u_{\lambda}\right)=c_{\lambda}^{M}$.

\section{Solution for Equation (1)}

In this section, we prove the main theorem. By the similar Moser iteration Lemma in $[21,22]$, we only need to prove the following lemma.

Lemma 5. There exist two constants $B, D>0$ independent on $m$ such that $\left|u_{0}\right|_{\infty} \leq B(1+\lambda)^{D}$.

Proof. Similar to (10), we can obtain the following:

$$
\begin{aligned}
4 c_{\lambda}^{M} & =4 I_{\lambda}^{M}\left(u_{0}\right)-\left\langle\left(I_{\lambda}^{M}\right)^{\prime}\left(u_{0}\right), u_{0}\right\rangle \\
& =\int_{\mathbb{R}^{N}}\left[a\left|\nabla u_{0}\right|^{2}+V(x)\left|u_{0}\right|^{2}\right] \mathrm{d} x+\int_{\mathbb{R}^{N}}\left(I_{\alpha} * F\left(u_{0}\right)\right)\left[f\left(u_{0}\right) u_{0}-2 F\left(u_{0}\right)\right] \mathrm{d} x+\lambda \int_{\mathbb{R}^{N}}\left[h_{M}\left(u_{0}\right) u_{n}-4 H_{M}\left(u_{0}\right)\right] \mathrm{d} x \\
& \geq \int_{\mathbb{R}^{N}}\left[a\left|\nabla u_{0}\right|^{2}+V(x)\left|u_{0}\right|^{2}\right] \mathrm{d} x+\lambda \int_{\mathbb{R}^{N}}\left[h_{M}\left(u_{0}\right) u_{0}-4 H_{M}\left(u_{0}\right)\right] \mathrm{d} x \\
& \geq \int_{\mathbb{R}^{N}}\left[a\left|\nabla u_{0}\right|^{2}+V(x)\left|u_{0}\right|^{2}\right] \mathrm{d} x-\lambda \int_{\mathbb{R}^{N}} \theta\left|u_{0}^{+}\right|^{2} \mathrm{~d} x .
\end{aligned}
$$

then, by similar argument as the proof of Lemma 3, we can know that $\left\|u_{0}\right\|$ is bounded and that there exists $Q>0$ such that $\left\|u_{0}\right\| \leq Q$.

Next, set $T>2, r>0$ and $\tilde{u}_{0}^{T}:=b\left(u_{0}\right)$, where $b: \mathbb{R} \rightarrow \mathbb{R}$ is a smooth function satisfying $b(s)=s$ for $|s| \leq T-1, b(-s)=-b(s) ; b^{\prime}(s)=0$ for $s \geq T$ and $b^{\prime}(s)$ is decreasing in $[T-1, T]$. This implies the following:

$$
\begin{cases}\tilde{u}_{0}^{T}=u_{0}, & \text { for }\left|u_{0}\right| \leq T-1, \\ \left|\tilde{u}_{0}^{T}\right|=\left|b\left(u_{0}\right)\right| \leq\left|u_{0}\right|, & \text { for } T-1 \leq\left|u_{0}\right| \leq T, \\ \left|\tilde{u}_{0}^{T}\right|=C_{T}>0, & \text { for }\left|u_{0}\right| \geq T,\end{cases}
$$

where $T-1 \leq C_{T} \leq T$. Moreover, one can easily obtain the following.

$$
0 \leq \frac{s b^{\prime}(s)}{b(s)} \leq 1, \forall s \neq 0
$$

Let $\psi=u_{0}\left|\tilde{u}_{0}^{T}\right|^{2 r}$. Then $\psi \in X$, hence by taking $\psi$ as the test function, one obtains the following.

$$
\begin{aligned}
\int_{\mathbb{R}^{N}}\left(I_{\alpha}\right. & \left.* F\left(u_{0}\right)\right) f\left(u_{0}\right) \psi \mathrm{d} x+\lambda \int_{\mathbb{R}^{N}} h_{M}\left(u_{0}\right) \psi \mathrm{d} x \\
& =a \int_{\mathbb{R}^{N}} \nabla u_{0} \nabla \psi \mathrm{d} x+b \int_{\mathbb{R}^{N}}\left|\nabla u_{0}\right|^{2} \mathrm{~d} x \int_{\mathbb{R}^{N}} \nabla u_{0} \nabla \psi \mathrm{d} x+\int_{\mathbb{R}^{N}} V(x) u_{0} \psi \mathrm{d} x .
\end{aligned}
$$


Note that the following obtains.

$$
\begin{aligned}
\int_{\mathbb{R}^{N}} \nabla u_{0} & \nabla \psi \mathrm{d} x \\
\geq & \int_{\left|u_{0}\right| \leq T-1}(1+r)\left|\tilde{u}_{0}^{T}\right|^{2 r}\left|\nabla u_{0}\right|^{2} \mathrm{~d} x+\int_{\left|u_{0}\right| \geq T}\left|\tilde{u}_{0}^{T}\right|^{2 r}\left|\nabla u_{0}\right|^{2} \mathrm{~d} x \\
& +\int_{T-1<\left|u_{0}\right|<T}\left[\left|\tilde{u}_{0}^{T}\right|^{2 r}+2 r u_{0} b\left(u_{0}\right) b^{\prime}\left(u_{0}\right)\left|\tilde{u}_{0}^{T}\right|^{2 r-2}\right]\left|\nabla u_{0}\right|^{2} \mathrm{~d} x \\
\geq & \int_{\left|u_{0}\right| \leq T-1}\left|\tilde{u}_{0}^{T}\right|^{2 r}\left|\nabla u_{0}\right|^{2} \mathrm{~d} x+\int_{\left|u_{0}\right| \geq T}\left|\tilde{u}_{0}^{T}\right|^{2 r}\left|\nabla u_{0}\right|^{2} \mathrm{~d} x \\
& +\int_{T-1<\left|u_{0}\right|<T}\left[\left|\tilde{u}_{0}^{T}\right|^{2 r}+2 r u_{0} b\left(u_{0}\right) b^{\prime}\left(u_{0}\right)\left|\tilde{u}_{0}^{T}\right|^{2 r-2}\right]\left|\nabla u_{0}\right|^{2} \mathrm{~d} x \\
\geq & \frac{1}{(1+r)^{2}} \int_{\left|u_{0}\right| \leq T-1}\left|\nabla\left[u_{0}\left(\tilde{u}_{0}^{T}\right)^{r}\right]\right|^{2} \mathrm{~d} x+\int_{\left|u_{0}\right| \geq T}\left|\nabla\left[u_{0}\left(\tilde{u}_{0}^{T}\right)^{r}\right]\right|^{2} \mathrm{~d} x \\
& +\int_{T-1<\left|u_{0}\right|<T}\left[\left|\tilde{u}_{0}^{T}\right|^{2 r}+2 r u_{0}^{2}\left(b^{\prime}\left(u_{0}\right)\right)^{2}\left|\tilde{u}_{0}^{T}\right|^{2 r-2}\right]\left|\nabla u_{0}\right|^{2} \mathrm{~d} x \\
\geq & \frac{1}{(1+r)^{2}} \int_{\left|u_{0}\right| \leq T-1}\left|\nabla\left[u_{0}\left(\tilde{u}_{0}^{T}\right)^{r}\right]\right|^{2} \mathrm{~d} x+\int_{\left|u_{0}\right| \geq T}\left|\nabla\left[u_{0}\left(\tilde{u}_{0}^{T}\right)^{r}\right]\right|^{2} \mathrm{~d} x \\
& +\int_{T-1<\left|u_{0}\right|<T}\left[\frac{1}{(1+r)^{2}}\left|\tilde{u}_{0}^{T}\right|^{2 r}+\frac{r}{(1+r)^{2}} 2 r u_{0}^{2}\left(b^{\prime}\left(u_{0}\right)\right)^{2}\left|\tilde{u}_{0}^{T}\right|^{2 r-2}\right]\left|\nabla u_{0}\right|^{2} \mathrm{~d} x \\
= & \frac{1}{(1+r)^{2}} \int_{\left|u_{0}\right| \leq T-1}\left|\nabla\left[u_{0}\left(\tilde{u}_{0}^{T}\right)^{r}\right]\right|^{2} \mathrm{~d} x+\int_{\left|u_{0}\right| \geq T}\left|\nabla\left[u_{0}\left(\tilde{u}_{0}^{T}\right)^{r}\right]\right|^{2} \mathrm{~d} x \\
& +\int_{T-1<\left|u_{0}\right|<T}\left[\frac{1}{(1+r)^{2}} b^{2 r}\left(u_{0}\right)\left|\nabla u_{0}\right|^{2}+\frac{2}{(1+r)^{2}} u_{0}^{2}\left|\nabla b^{r}\left(u_{0}\right)\right|^{2}\right] \mathrm{d} x \\
\geq & \frac{1}{(1+r)^{2}} \int_{\left|u_{0}\right| \leq T-1}\left|\nabla\left[u_{0}\left(\tilde{u}_{0}^{T}\right)^{r}\right]\right|^{2} \mathrm{~d} x+\int_{\left|u_{0}\right| \geq T}\left|\nabla\left[u_{0}\left(\tilde{u}_{0}^{T}\right)^{r}\right]\right|^{2} \mathrm{~d} x \\
& +\frac{2 C_{1}}{(1+r)^{2}} \int_{T-1<\left|u_{0}\right|<T}\left[b^{2 r}\left(u_{0}\right)\left|\nabla u_{0}\right|^{2}+u_{0}^{2}\left|\nabla b^{r}\left(u_{0}\right)\right|^{2}\right] \mathrm{d} x \\
\geq & \frac{1}{(1+r)^{2}} \int_{\left|u_{0}\right| \leq T-1}\left|\nabla\left[u_{0}\left(\tilde{u}_{0}^{T}\right)^{r}\right]\right|^{2} \mathrm{~d} x+\int_{\left|u_{0}\right| \geq T}\left|\nabla\left[u_{0}\left(\tilde{u}_{0}^{T}\right)^{r}\right]\right|^{2} \mathrm{~d} x \\
& +\frac{C_{1}}{(1+r)^{2}} \int_{T-1<\left|u_{0}\right|<T}\left|\nabla\left[u_{0}\left(\tilde{u}_{0}^{T}\right)^{r}\right]\right|^{2} \mathrm{~d} x \\
& \\
& \\
& \\
&
\end{aligned}
$$

Hence, by (14), we obtain the following.

$$
\begin{aligned}
\int_{\mathbb{R}^{N}}\left(I_{\alpha} *\left|u_{0}\right|^{p}\right)\left|u_{0}\right|^{p}\left|\tilde{u}_{0}^{T}\right|^{2 r} \mathrm{~d} x+\lambda \int_{\mathbb{R}^{N}} h_{M}\left(u_{0}\right) u_{0}\left|\tilde{u}_{0}^{T}\right|^{2 r} \mathrm{~d} x \\
\quad \geq \frac{C_{1}}{(1+r)^{2}} \int_{\mathbb{R}^{N}}\left|\nabla\left[u_{0}\left(\tilde{u}_{0}^{T}\right)^{r}\right]\right|^{2} \mathrm{~d} x+\int_{\mathbb{R}^{N}} V(x)\left|u_{0}\right|^{2}\left|\tilde{u}_{0}^{T}\right|^{2 r} \mathrm{~d} x .
\end{aligned}
$$

For any $\varepsilon>0$, by properties of $\tilde{u}_{m}^{T}$ and $h_{M}$, there exists $C_{\varepsilon}>0$ such that: $\int_{\mathbb{R}^{N}}\left(I_{\alpha} * F\left(u_{0}\right)\right) f\left(u_{0}\right) u_{0}\left|\tilde{u}_{0}^{T}\right|^{2 r} \mathrm{~d} x \leq T_{1} \int_{\mathbb{R}^{N}}\left(I_{\alpha} * F\left(u_{0}\right)\right) f\left(u_{0}\right) u_{0} \mathrm{~d} x \leq T_{2}\left(\|u\|^{\frac{2 N+2 \alpha}{N}}+\|u\|^{\frac{2 N+2 \alpha}{N-2}}\right) \leq M_{0}$.

where $T_{1}, T_{2}$ and $M_{0}$ are positive constants and the following applies.

$$
\left|h_{M}(t)\right| \leq C_{M}^{\prime}|t|+C_{M}|t|^{2^{*}-1}
$$

For all $t \in \mathbb{R}$. Therefore, for fixed $\lambda>0$ and small $\varepsilon>0$, we can deduce the following: 


$$
\begin{aligned}
\frac{C_{1}}{(1+r)^{2}} & \int_{\mathbb{R}^{N}}\left|\nabla\left[u_{0}\left(\tilde{u}_{0}^{T}\right)^{r}\right]\right|^{2} \mathrm{~d} x \\
& \leq \int_{\mathbb{R}^{N}}\left(I_{\alpha} * F\left(u_{0}\right)\right) f\left(u_{0}\right) u_{0}\left|\tilde{u}_{0}^{T}\right|^{2 r} \mathrm{~d} x+\lambda \int_{\mathbb{R}^{N}} h_{M}\left(u_{0}\right) u_{0}\left|\tilde{u}_{0}^{T}\right|^{2 r} \mathrm{~d} x-\int_{\mathbb{R}^{N}} V(x)\left|u_{0}\right|^{2}\left|\tilde{u}_{0}^{T}\right|^{2 r} \mathrm{~d} x \\
& \leq M+\int_{\mathbb{R}^{N}} V_{0}\left|u_{0}\right|^{2}\left|\tilde{u}_{0}^{T}\right|^{2 r} \mathrm{~d} x+\lambda C \int_{\mathbb{R}^{N}} u_{0}^{p}\left|\tilde{u}_{0}^{T}\right|^{2 r} \mathrm{~d} x-\int_{\mathbb{R}^{N}} V_{0}\left|u_{0}\right|^{2}\left|\tilde{u}_{0}^{T}\right|^{2 r} \mathrm{~d} x \\
& \leq(1+\lambda) C \int_{\mathbb{R}^{N}} u_{0}^{p}\left|\tilde{u}_{0}^{T}\right|^{2 r} \mathrm{~d} x .
\end{aligned}
$$

Notice that the following is the case.

$$
\frac{C_{2}}{(1+r)^{2}}\left[\int_{\mathbb{R}^{N}}\left|u_{0}\right|^{2^{*}}\left|\tilde{u}_{0}^{T}\right|^{2 r \cdot 2^{*}} \mathrm{~d} x\right]^{\frac{2}{2^{*}}} \leq \frac{C_{1}}{(1+r)^{2}} \int_{\mathbb{R}^{N}}\left|\nabla\left[u_{0}\left(\tilde{u}_{0}^{T}\right)^{r}\right]\right|^{2} \mathrm{~d} x
$$

Consequently, the following is obtained.

$$
\left[\int_{\mathbb{R}^{N}}\left|u_{0}\right|^{2^{*}}\left|\tilde{u}_{0}^{T}\right|^{2 r \cdot \frac{2^{*}}{2}} \mathrm{~d} x\right]^{\frac{2}{2^{*}}} \leq(1+\lambda) C(r+1)^{2} \int_{\mathbb{R}^{N}} u_{0}^{2^{*}}\left|\tilde{u}_{0}^{T}\right|^{2 r} \mathrm{~d} x .
$$

Take $r_{0}>0$ and $r_{k}=r_{0}\left(\frac{2^{*}}{2}\right)^{k}=r_{k-1} \cdot \frac{2^{*}}{2}$. Then,

$$
\begin{aligned}
{\left[\int_{\mathbb{R}^{N}} \mid\right.} & \left.\left.u_{0}\right|^{2^{*}}\left|\tilde{u}_{0}^{T}\right|^{2 r_{k}} \mathrm{~d} x\right]^{\frac{1}{2 r_{k}}} \\
\leq & {\left[\sqrt{1+\lambda} \sqrt{C}\left(r_{k-1}+1\right)\right]^{\frac{1}{r_{k-1}}}\left[\int_{\mathbb{R}^{N}}\left|u_{0}\right|^{2^{*}}\left|\tilde{u}_{0}^{T}\right|^{2 r_{k-1}} \mathrm{~d} x\right]^{\frac{1}{2 r_{k-1}}} } \\
\leq & \prod_{i=0}^{k-1}\left[\sqrt{1+\lambda} \sqrt{C}\left(r_{i}+1\right)\right]^{\frac{1}{r_{i}}}\left[\int_{\mathbb{R}^{N}}\left|u_{0}\right|^{2^{*}}\left|\tilde{u}_{0}^{T}\right|^{2 r_{0}} \mathrm{~d} x\right]^{\frac{1}{2 r_{0}}} \\
\quad & \prod_{i=0}^{k-1}(1+\lambda)^{\frac{1}{2 r_{i}}} \prod_{i=0}^{k-1}\left[\sqrt{C}\left(r_{i}+1\right)\right]^{\frac{1}{r_{i}}}\left[\int_{\mathbb{R}^{N}}\left|u_{0}\right|^{2^{*}}\left|\tilde{u}_{0}^{T}\right|^{2 r_{0}} \mathrm{~d} x\right]^{\frac{1}{2 r_{0}}} \\
& =\prod_{i=0}^{k-1}(1+\lambda)^{\frac{1}{2 r_{i}}} \exp \left\{\sum_{i=0}^{k-1} \frac{1}{r_{i}} \ln \left[\sqrt{C}\left(r_{i}+1\right)\right]\right\}\left[\int_{\mathbb{R}^{N}}\left|u_{0}\right|^{2^{*}}\left|\tilde{u}_{0}^{T}\right|^{2 r_{0}} \mathrm{~d} x\right]^{\frac{1}{2 r_{0}}} .
\end{aligned}
$$

Notice that the following is the case:

$$
\begin{aligned}
& {\left[\int_{\mathbb{R}^{N}}\left|u_{0}\right|^{2^{*}}\left|\tilde{u}_{0}^{T}\right|^{2 r_{0} \cdot \frac{N}{N-2}} \mathrm{~d} x\right]^{\frac{N-2}{N}}} \\
& \quad \leq C\left(r_{0}+1\right)^{2} \int_{\mathbb{R}^{N}}\left|u_{0}\right|^{2^{*}}\left|\tilde{u}_{0}^{T}\right|^{2 r_{0}} \mathrm{~d} x \\
& \quad \leq C\left(r_{0}+1\right)^{2} \int_{\left|u_{0}(x)\right|<\rho}\left|u_{0}\right|^{2^{*}}\left|\tilde{u}_{0}^{T}\right|^{2 r_{0}} \mathrm{~d} x+ \\
& \quad C\left(r_{0}+1\right)^{2}\left(\int_{\left|u_{0}(x)\right| \geq \rho}\left|u_{0}\right|^{2^{*}} \mathrm{~d} x\right)^{\frac{2}{N}}\left(\int_{\mathbb{R}^{N}}\left|u_{0}\right|^{2^{*}}\left|\tilde{u}_{0}^{T}\right|^{2 r_{0} \cdot \frac{N}{N-2}} \mathrm{~d} x\right)^{\frac{N-2}{N}} .
\end{aligned}
$$

Take $\rho>0$ to be such that:

$$
C\left(r_{0}+1\right)^{2}\left(\int_{\left|u_{0}(x)\right| \geq \rho}\left|u_{0}\right|^{2^{*}} \mathrm{~d} x\right)^{\frac{2}{N}}<\frac{1}{2} .
$$


Then the following obtains:

$$
\left[\int_{\mathbb{R}^{N}}\left|u_{0}\right|^{2^{*}}\left|\tilde{u}_{0}^{T}\right|^{2 r_{0} \cdot \frac{N}{N-2}} \mathrm{~d} x\right]^{\frac{N-2}{N}} \leq C\left(r_{0}+1\right)^{2} \int_{\left|u_{0}(x)\right|<\rho}\left|u_{0}\right|^{2^{*}}\left|\tilde{u}_{0}^{T}\right|^{2 r_{0}} \mathrm{~d} x \leq C .
$$

Set the following:

$$
d_{k}=\prod_{i=0}^{k-1}\left[\sqrt{C}\left(r_{i}+1\right)\right]^{\frac{1}{r_{i}}}=\exp \left\{\sum_{i=0}^{k-1} \frac{1}{r_{i}} \ln \left[\sqrt{C}\left(r_{i}+1\right)\right]\right\}
$$

and:

$$
e_{k}=\prod_{i=0}^{k-1}(1+\lambda)^{\frac{1}{2 r_{i}}}=(1+\lambda)^{\frac{2^{*}}{\left(2^{*}-2\right) 2 r_{0}}\left[1-\left(\frac{2}{2^{*}}\right)^{k}\right]}
$$

Then $d_{k} \rightarrow d_{\infty}$ as $k \rightarrow \infty$ and $e_{k} \rightarrow e_{\infty}=(1+\lambda)^{\frac{2^{*}}{\left(2^{*}-2\right) 2 r_{0}}}$ as $k \rightarrow \infty$. By (15), we know that the following is the case.

$$
\begin{aligned}
& {\left[\int_{\mathbb{R}^{N}}\left|u_{0}\right|^{2^{*}}\left|\tilde{u}_{0}^{T}\right|^{2 r_{k}} \mathrm{~d} x\right]^{\frac{1}{2 r_{k}}}} \\
& \quad \leq d_{k} e_{k}\left[\int_{\mathbb{R}^{N}}\left|u_{0}\right|^{2^{*}}\left|\tilde{u}_{0}^{T}\right|^{2 r_{0}} \mathrm{~d} x\right]^{\frac{1}{2 r_{0}}} \\
& \quad \leq d_{k} e_{k}\left[\left(\int_{\mathbb{R}^{N}}\left|u_{0}\right|^{2^{*}} \mathrm{~d} x\right)^{\frac{2}{N}}\left(\int_{\mathbb{R}^{N}}\left|u_{0}\right|^{2^{*}}\left|\tilde{u}_{0}^{T}\right|^{2 r_{0} \cdot \frac{N}{N-2}} \mathrm{~d} x\right)^{\frac{N-2}{N}}\right]^{\frac{1}{2 r_{0}}} \\
& \quad \leq C d_{k} e_{k}\left(\int_{\mathbb{R}^{N}}\left|u_{0}\right|^{2^{*}} \mathrm{~d} x\right)^{\frac{1}{N r_{0}}} \leq C d_{k} e_{k} .
\end{aligned}
$$

From (16), by Fatou Lemma with $T \rightarrow+\infty$, one has the following.

$$
\left|u_{0}\right|_{2^{*}+2 r_{k}}^{\frac{2^{*}+2 r_{k}}{2 r_{k}}} \leq C d_{k} e_{k}
$$

Consequently, let $k \rightarrow \infty$ and we obtain the following:

$$
\left|u_{0}\right|_{\infty} \leq C d_{\infty} e_{\infty}=C d_{\infty}(1+\lambda)^{\frac{2^{*}}{\left(2^{*}-2\right) 2 r_{0}}}:=B(1+\lambda)^{D},
$$

where $B>0$ and $D>0$. Thus we complete the proof.

Proof of Theorem 1. By Lemma 5, for large $M>0$, we can choose small $\lambda_{0}>0$ such that $\left|u_{0}\right|_{\infty} \leq B(1+\lambda)^{D} \leq M$ for all $\lambda \in\left(0, \lambda_{0}\right]$. The consequence $u_{0}$ is also a nontrivial solution of Equation (1) with $\lambda \in\left(0, \lambda_{0}\right]$. Thus, we complete the proof.

Author Contributions: Conceptualization, L.Z. and C.Z.; methodology, L.Z.; software, L.Z.; validation, L.Z.; formal analysis, L.Z.; investigation, L.Z.; resources, C.Z.; data curation, L.Z.; writingoriginal draft preparation, L.Z.; writing—review and editing, L.Z. and C.Z.; supervision,C.Z.; project administration, C.Z.; funding acquisition, C.Z. All authors have read and agreed to the published version of the manuscript.

Funding: This research was funded by the National Natural Science Foundation of China grant number No. 11771198, 11361042, 11901276)

Institutional Review Board Statement: Not applicable.

Informed Consent Statement: Not applicable.

Data Availability Statement: Not applicable. 
Conflicts of Interest: The authors declare no conflicts of interest.

\section{References}

1. Li, G.; Ye, H. Existence of positive ground state solutions for the nonlinear Kirchhoff type equations in $\mathbb{R}^{3}$. J. Differ. Equations 2014, 257, 566-600. [CrossRef]

2. Chen, C.; Kuo, Y.; Wu, T. The Nehari manifold for a Kirchhoff type problem involving sign-changing weight functions. J. Differ Equations 2011, 250, 1876-1908. [CrossRef]

3. He, X.; Zou, W.M. Existence and concentration behavior of positive solutions for a Kirchhoff equation in $\mathbb{R}^{3}$. J. Differ. Equations 2012, 252, 1813-1834. [CrossRef]

4. Guo, Z. Ground states for Kirchhoff equations without compact condition. J. Differ. Equations 2015, 259, 2884-2902. [CrossRef]

5. Lü, D. A note on Kirchhoff-type equations with Hartree-type nonlinearities. Nonlinear Annal. 2014, 99, 35-48. [CrossRef]

6. Chen, P.; Liu, X.C. Ground states for Kirchhoff equation with Hartree-type nonlinearities. J. Math. Anal. Appl. 2019, 473, 587-608. [CrossRef]

7. Ye, H. Positive high energy solution for Kirchhoff equation in $\mathbb{R}^{3}$ with superlinear nonlinearities via Nehari-Pohozaev manifold Discrete Contin. Dyn. Syst. 2015, 35, 3857-3877. [CrossRef]

8. Li, Y.; Li, F.; Shi, J. Existence of a positive solution to Kirchhoff type problems without compactness conditions. J. Differ. Equations 2012, 253, 2285-2294. [CrossRef]

9. Li, Y.; Li, F.; Shi, J. Existence of positive solutions to Kirchhoff type problems with zero mass. J. Math. Anal. Appl. 2014, 410, 361-374. [CrossRef]

10. Li, Y.H.; Geng, Q. The existence of nontrivial solution to a class of nonlinear Kirchhoff equations without any growth and Ambrosetti-Rabinowitz. Appl. Math. Lett. 2019, 96, 153-158. [CrossRef]

11. Figueiredo, G.M.; Morales-Rodrigo, C. Santos Júnior, J.; Suárez, A. Study of a nonlinear Kirchhoff equation with non-homogeneous material. J. Math. Anal. Appl. 2014, 416, 597-608. [CrossRef]

12. Moroz, I.M.; Penrose, R.; Tod, P. Spherically-symmetric solutions of Schrödinger-Newton equations. Class. Quantum Gravity 1998, 15, 2733-2742. [CrossRef]

13. Moroz, V.; van Schaftingen, J. Ground states of nonlinear Choquard equations: existence, qualitative properties and decay asymptotics. J. Funct. Anal. 2013, 265, 153-184. [CrossRef]

14. Moroz, V.; van Schaftingen, J. Existence of ground states for a class of nonlinear Choquard equations. Trans. Amer. Math. Soc. 2015, 367, 6557-6579. [CrossRef]

15. Moroz, V.; van Schaftingen, J. Nonexistence and optimal decay of supersolutions to Choquard equations in exterior domains. $J$. Differ. Equations 2013, 254, 3089-3145. [CrossRef]

16. Moroz, V.; van Schaftingen, J. Ground states of nonlinear Choquard equations: Hardy-Littlewood-Sobolev critical exponent. Commun. Contemp. Math. 2015, 17, 1550005. [CrossRef]

17. Moroz, V.; van Schaftingen, J. A guide to the Choquard equation. J. Fixed Point Theory Appl. 2017, 19, 773-813. [CrossRef]

18. Chimenti, M.; van Schaftingen, J. Nodal solutions for the Choquard equation. J. Funct. Anal. 2016, 271, 107-135. [CrossRef]

19. Ma, L.; Lin, Z. Classification of positive solitary solutions of the nonlinear Choquard equation. Arch Ration. Mech. Aral. 2010, 195, 455-467. [CrossRef]

20. Li, G.D.; Li, Y.Y.; Tang, C.L.; Yin, L.F. Existence and concentrate behavior of ground state solutions for critical Choquard equations. Appl. Math. Lett. 2019, 96, 101-107. [CrossRef]

21. Li, Q.; Teng, K.M.; Wu, X. Ground states for Kirchhoff-type equations with critical or supercritical growth. Math. Meth. Appl. Sci. 2017, 40, 6732-6746. [CrossRef]

22. Li, Q.; Teng, K.M.; Wu, X. Existence of nontrivial solutions for Schrödinger-Kirchhoff type equations with critical or supercritical growth. Math. Meth. Appl. Sci. 2018, 41, 1136-1144. [CrossRef]

23. Bartsch, T.; Wang, Z.Q. Existence and multiplicity results for some superlinear elliptic problems on $\mathbb{R}^{N}$. Comm. Partial. Differ. Equations 1995, 20, 1725-1741. [CrossRef]

24. Lieb, E.H.; Loss, M. Analysis, 2nd ed.; Grad. Stud. Math. ; American Mathematical Society: Providence, RL, USA, 2001 ; Volume 14.

25. Willem, M. Minimax Theorems, Progress in Nonlinear Differential Equations and Their Applications 24; Birkhäuser: Boston, MA, USA, 1996. 